\title{
A Rare Image of Liver Steatosis - Acid Lipase Lysosomal Deficiency
}

\author{
Ioana Călian ${ }^{1}$, Ioana Rusu ${ }^{1,2}$, Cristina Sabo ${ }^{2,3}$, Lidia Ciobanu $^{1,2}$
}

1) Regional Institute of Gastroenterology and Hepatology, Cluj-Napoca; 2) Iuliu Hatieganu University of Medicine and Pharmacy, Cluj-Napoca; 3) $2^{\text {nd }}$ Medical Department, Cluj-Napoca, Romania
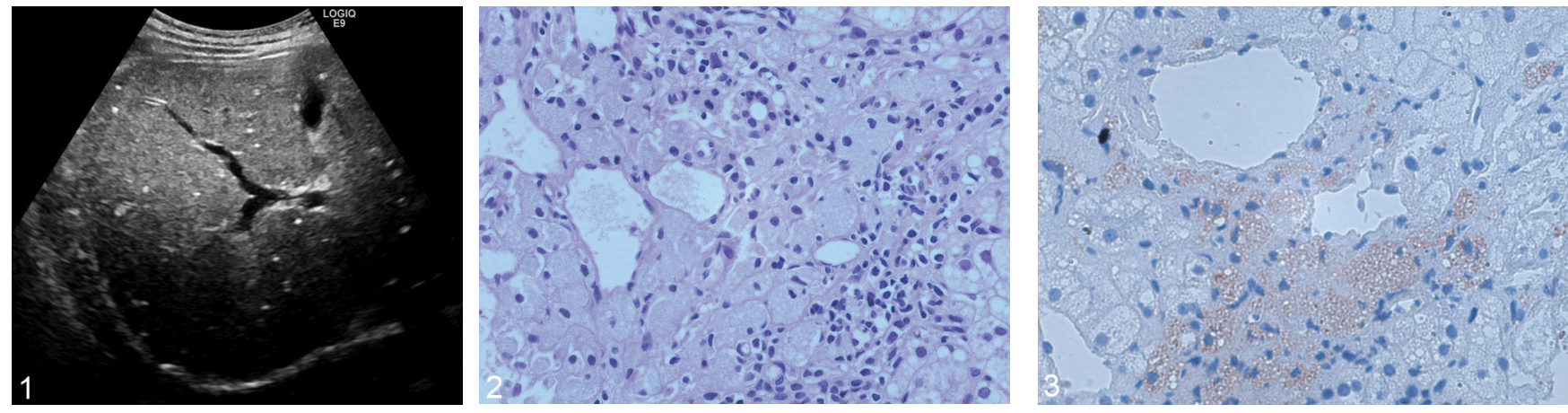

Lysosomal acid lipase deficiency (LAL-D) is a rare lysosomal storage disease. Milder forms in adolescents and young adults frequently go undiagnosed for prolonged periods. These patients frequently present with progressive fatty liver disease, enlarged spleen, atherogenic dyslipidemia and premature atherosclerosis [1].

We present the case of an 18-year old female investigated for hepatosplenomegaly for more than 5 years in different medical centres. She had normal body weight and persistent high levels of transaminases, associated with high levels of cholesterol. Viral hepatitis, autoimmune hepatitis, Wilson disease and juvenile hemochromatosis had been previously excluded by serological tests. On ultrasound the liver parenchyma was hyperechoic with small hyperechoic dots, distributed in both lobes, so called "starry sky liver" (Fig. 1). The spleen was enlarged and homogeneous. There were no signs of portal hypertension. Liver biopsy detected hepatocytes with micro-vesicular steatosis, clusters of foamy histiocytes with light tan-coloured cytoplasm and pyknotic nuclei (Fig. 2, H\&E 400X) within the sinusoids and portal tracts. The foamy macrophages contained cholesterol and/or cholesterol esters, depicted by Oil Red staining (Fig. 3, 400x). This aspect was suggestive of LAL-D, further confirmed by serological enzymatic test (acid lipase activity less than $0.02 \mathrm{nmol} / \mathrm{punch} /$ hour). The genetic testing for mutations in LIPA gene detected the presence of a heterozygous pathogenic variant: c.894G>A (p.Gln298Gln) and another classified as likely pathogenic: C.309C >A (p.Ser103Arg).

According to all guidelines, even though it is rare, LAL-D should be considered in lean young adults with non-alcoholic fatty liver disease with micro-vesicular steatosis on liver biopsy [2]. For clinicians, before liver biopsy, the ultrasound peculiar aspect of "starry sky liver" on increased liver echogenicity should be a "red flag" to consider LAL-D. The "starry sky appearance" is frequently associated with acute hepatitis, but with poor sensitivity and specificity [3]. In LAL-D on a hyperechoic texture of liver parenchyma, the bright dots might be explained by the clusters of foamy macrophages within the portal tracts. This is the first Romanian reported case of LAL-D.

Corresponding author: Ioana Rusu, ioanarussu@yahoo.com

Conflicts of interest: None to declare.

Acknowledgments: We thank Tudor Lucian Pop for enzymatic tests.

\section{REFERENCES}

1. Strebinger G, Müller E, Feldman A, Aigner E. Lysosomal acid lipase deficiency - early diagnosis is the key. Hepat Med 2019;11:79-88. doi:10.2147/HMER.S201630

2. European Association for the Study of the Liver (EASL); European Association for the Study of Diabetes (EASD); European Association for the Study of Obesity (EASO). EASL-EASD-EASO Clinical Practice Guidelines for the management of non-alcoholic fatty liver disease. J Hepatol 2016;64:1388-1402. doi:10.1016/j.jhep.2015.11.004

3. Giorgio A, Amoroso P, Fico P, et al. Ultrasound evaluation of uncomplicated and complicated acute viral hepatitis. J Clin Ultrasound 1986;14:675-679. doi:10.1002/jcu.1870140903 